\title{
Silicate Granules Preconditioned with Human Bone Marrow Mononuclear Cells Improve Osteogenesis in Bone Sarcoma Patients
}

Flavio Fazioli ${ }^{2}$, Gianluca Colella ${ }^{3}$, Rosa Camerlingo ${ }^{4}$, Roberta Miceli ${ }^{4}$, Mariano Giuseppe Di Salvatore ${ }^{2}$, Giuseppe Pirozzi ${ }^{4}$ and Filomena de Nigris ${ }^{1 *}$

${ }^{1}$ Department of Biochemistry Biophysics and General Pathology Second University of Naples, Naples, Italy

${ }^{2}$ Division of Musculoskeletal Oncology Surgery, National Cancer Institute, Pascale Foundation, Naples, Italy

${ }^{3}$ Department of Human Health, Federico II University of Naples, Naples, Italy

${ }^{4}$ Department of Sperimental Oncology, National Cancer Institute, Pascale Foundation, Naples, Italy

\section{Abstract}

Background: Poor bone regeneration is a devastating complication after large tumor resection. Different synthetic bone-grafting materials have been used to restore skeletal defects. Several studies evaluated the capability of mesenchymal stem cells to improve the efficacy of bone regenerative potential of biomaterials. However, there are no data, in human, about the combinatory use of silicate granules with autologous Bone Marrow Mononuclear cells (BMMCs) to refill bone cavity.

Methods: BMMCs were prepared in accordance with the International Society for Cell Therapy guidelines. MTT assays and scanning electron microscopy evaluated the biocompatibility, and adhesion of BMMCs to the granular silicate bone substitute. In vivo study is based on twenty patients with malignant osteolytic lesions. The mean volume of the lesions, measured by pre-operative computed tomography (CT) was $18.5 \mathrm{~cm}^{2}$ (range 16-24 $\mathrm{cm} 2$ ). Sixteen patients were treated post-surgery with curettage and refilling with granular silicate alone, and four with a combination of granular silicate and BMMCs The follow-up was determined by clinical, Musculoskeletal Tumor Society functional state score system (MSTS), and radiograph examination, moreover, the callus type was classified according to the International System.

Results: Scanning electron microscopy showed that $90 \%$ of BMMCs adhered to silicate granules in a few minutes and long pseudopodia contacting extra-cellular matrix. Patients treated with autologous BMMCs and granular silicate developed bone callus after two weeks. The follow-up of limbs functional state measured by MSTS was a mean of $82 \%$ compared to mean of $60 \%$ obtained with granules alone. At the end of the follow-up (minimum one year), all the patients were cancer-free with an excellent outcome.

Conclusions: The encouraging results of our early study indicate that refilling at the osteotomy site with autologous BMMCs and granular silicate improves bone repair. A larger study cohort and longer follow-up times are required to identify additional predictors and indications.

Keywords: Sarcomas; Angiogenesis; Endothelial cells; Tumor microenvironment

\section{Introduction}

The surgical resection of bone tumors often leads to osseous nonunions and loss of structural integrity [1]. Different strategies have been used to repair and restore skeletal defects, with good mechanical and functional properties [2-8]. The role of synthetic and bone-graft substitutes is not only to replace missing bone but also to encourage bone integration, i.e., by acting as a scaffold to guide bone growth into the graft [9]. Technological advances along with a better understanding of bone-healing biology have led to the development of various porous ceramics that are currently available to orthopedic surgeons [10]. Threedimensional (3D) porous materials are therefore preferentially used to provide a better environment for cellular attachment and proliferation, and their architecture defines the ultimate shape of new bone [11]. One of the most successful synthetic bone-grafting materials is Actifuse ${ }^{\circ}$ (Baxter Healthcare, Newbury, UK), made up of HA-substituted porous silicon granules $(2-5 \mathrm{~mm})[7,11]$. Actifuse ${ }^{\oplus}$ granules bind bone via the rapid formation of a Hydroxycarbonate Apatite (HCA) surface layer and stimulates osteogenesis via their dissolution following osteoblast adhesion [8]. Currently, there is great interest in applications of biologic stimuli to enhance bone regeneration $[12,13]$. Several studies evaluated the capability of mesenchymal stem cells, with different origins, to improve the efficacy of bone regenerative potential of biomaterials in both animal models [14-16] and clinical settings [17-19]. Bone regeneration by autologous cell transplantation is one of the most promising treatment [17]. Mesenchymal stem cells (MSC) combined with an osteoconductive scaffold have been shown to support bone repair for bone tissue regeneration [20,21]. The main advantage in using cells as biological stimuli is due to autogenous properties. Their application can prevent possible complications, such as immunogenic reactions and disease transmissions, and are easy to prepare. The aim of the present study was to evaluate in vitro the biocompatibility, colonization and adhesion of Bone Marrow Mononuclear Cells (BMMCs) to silicate granules. Additionally, we report our findings on the use of silicate granules mixed with BMMCs in bone sarcoma patients to improve bone reconstruction.

*Corresponding author: Filomena de Nigris, Department of Biochemistry Biophysics and General Pathology Second University of Naples, Naples, Italy, Tel: 390815665686; E-mail: filomena.denigris@unina2.it

Received October 16, 2015; Accepted October 31, 2015; Published November 09, 2015

Citation: Fazioli F, Colella G, Camerlingo R, Miceli R, Salvatore MGD, et al. (2015) Silicate Granules Preconditioned with Human Bone Marrow Mononuclear Cells Improve Osteogenesis in Bone Sarcoma Patients. J Cancer Sci Ther 7: 321-327. doi:10.4172/1948-5956.1000370

Copyright: @ 2015 Fazioli F, et al. This is an open-access article distributed under the terms of the Creative Commons Attribution License, which permits unrestricted use, distribution, and reproduction in any medium, provided the original author and source are credited. 
Citation: Fazioli F, Colella G, Camerlingo R, Miceli R, Salvatore MGD, et al. (2015) Silicate Granules Preconditioned with Human Bone Marrow Mononuclear Cells Improve Osteogenesis in Bone Sarcoma Patients. J Cancer Sci Ther 7: 321-327. doi:10.4172/1948-5956.1000370

\section{Methods}

\section{Biomaterials}

Porous silicate substituted HA Actifuse ${ }^{\circledast}(0.8$ wt. \% Si) purchased from Baxter Healthcare, Newbury, UK) is composed by granules with sizes typically between 1 and $3 \mathrm{~mm}$. It was prepared with technology based on slurry expansion (following instruction procedures). Briefly, slurry with a high powder concentration was used and expanded in a known volume to achieve a total porosity of $80 \%$ in volume, which corresponds to a large surface area $\left(0.9 \mathrm{~m}^{2} / \mathrm{g}\right)$; its porosity is characterized by bi-modal porous structures and controlled morphology. Demineralized bovine bone matrix (DBM) particles are obtained from bovine cortical bone with sizes between 1 and $3 \mathrm{~mm}$. Their porosity equals natural human bone-micropores with a diameter of 1-2 $\mathrm{m}$ that were frequently seen. The DBM particles are sterilized by a validated per-acetic acid-based process (data published by the manufacturer (LifeNet health Italy).

\section{Isolation and growth of BMMSCs}

Iliac crest bone marrow aspirates $(20 \mathrm{ml})$ were obtained from four orthopedic patients under general anesthesia according to the ethical committee of the Istituto Nazionale Tumori G. Pascale Napoli Italy. All donors provided informed consent. Bone marrow mononuclear fraction was isolated by Ficoll-mediated (Histopaque, 10771, Sigma Company, Milan, Italy). Briefly, bone marrow aspirate was diluted in Hanks balanced salt solution (HBSS) to make the volume up to $30 \mathrm{ml}$. Cell solution was, gently overlaid on $60 \mathrm{ml}$ of Histopaque. The layer at the interface of the Ficoll and HBSS was collected after $30 \mathrm{~min}$ of centrifugation at $1,800 \mathrm{~g}$ at room temperature without brake. The interface cell layer was transferred and cell suspension was centrifuged at $1,000 \mathrm{~g}$ for $10 \mathrm{~min}$ at room temperature. The pellet was suspended and cells were seeded in a T75 flask and cultured with aMEM (Lonza, Milan, Italy) supplemented with $20 \%$ fetal bovine serum (FBS), 100 units $/ \mathrm{mL}$ penicillin (Euroclone, Wetherby, UK), $100 \mathrm{mg} / \mathrm{ml}$ streptomycin, and 1\% GlutaMAX (Gibco Invitrogen, Paisley, Scotland), and incubated at $37^{\circ} \mathrm{C}$ with $5 \% \mathrm{CO}_{2}$ in a humidified atmosphere.

\section{FACS analysis of BMMCs}

BMMCs detached from silicate granules (by non-enzymatic system GIBCO) were characterized by flow cytometry for specific surface antigens, in accordance with the International Society for Cell Therapy [26]. $1 \times 10^{5}$ cells was analyzed by FACS following incubation for $30 \mathrm{~min}$ at $4^{\circ} \mathrm{C}$ with specific phycoerythrin (PE) or fluorescein isothiocyanate (FITC) conjugated-antibodies or with the relevant control in PBS containing 2\% BSA. The following markers were assayed: CD44 (clone\#2F10 from R\&D System), CD45 (Bioscience, clone \#HI30), human Stro-1 (cat. 340106 BioLegend,), CD73 (Ecto50-nucleotidase, Biosciences clone \#AD2), CD90 (Thy-1, clone \#5E10), CD105 (Endoglin, Bioscience clone IgG1 SN6) CD29 (clone 4G7-2E3 form R\&D system), CD11b (Mouse IgG2b Clone \#238446), CD34 (Clone \#756510) CD14 (Mouse IgG1 Clone \#134620) from R\&D systems and CD 235 (clone\#HI264 from BioLegend). FACS AriaII (Becton Dickinson) was used to analyze and 30,000 cell events at each experimental point. Control experiments included incubation with isotopic human IgG (Becton Dickinson). Data were analyzed using FACS DIVA software (Becton Dickinson).

\section{Seeding of bone marrow mononuclear cells}

Plates were incubated with a mix of $1 \mathrm{ml}$ of slurry Actifuse ${ }^{\bullet}$ granules or demineralized bovine bone in $1 \mathrm{~mL}$ fibronectin solution (10 g/
$\mathrm{mL}$, Sigma, Deisenhofen, Germany) in PBS without $\mathrm{Mg}^{2+}$ and $\mathrm{Ca}^{2+}$ (PBS-l-) for $30 \mathrm{~min}$, forming a dense monolayer. The supernatants were removed and the granules were air-dried under sterile conditions at room temperature. Then, $2 \times 10^{4}$ cells/well were seeded into 24 -well pre-coated plates in EGM-2 containing 0.5\% Fetal Calf Serum plus growth factors (Lonza). After different times of incubation under the appropriate conditions, the medium containing the non-adhering cells was removed and rinsed once again over the bone-graft layer. This procedure was repeated three times. The (DBM and granules) were then gently transferred to another well. The remaining cells in the supernatant together with the bottom of the initial seeding well were collected and spin at 1,500 $\mathrm{g}$ for $5 \mathrm{~min}$. Cells were stained with $0.75 \%$ crystal violet in a solution of $50 \%$ ethanol, $0.25 \% \mathrm{NaCl}$, and $1.75 \%$ formaldehyde. Absorbance was read at $595 \mathrm{~nm}$ with an ELISA reader (Infinite M200, Tecan, Mainz, Germany). The percentage of adherent cells was calculated: [(initial cell number-remaining cell number)/initial cell number $] \times 100 \%$. All experiments were performed in triplicate.

\section{MTT assay}

For determination of the metabolic activity of the seeded cells the Cell Proliferation Kit I (MTT, Roche Diagnostics, Mannheim, Germany) was applied. The assay is based on the cleavage of the yellow tetrazolium salt MTT (3-[4,5-dimethylthiazol-2-yl]-2,5diphenyltetrazoliumbromide) to purple formazan crystals by metabolic active cells. BMMCs were seeded on a bone-graft substitutes monolayer covering in 24-well plate as described above. Before the addition of the MTT reagent the granules were transferred to an empty well in order to prevent false positive results caused by cells adhering to the bottom of the well. Ninety $\mu \mathrm{l}$ medium and $10 \mu \mathrm{l}$ of MTT labelling reagent were added to each well and cells were incubated additional $4 \mathrm{~h}$. Next, the cells were incubated overnight with a solubilization solution. The supernatants were collected and transferred to another 96-well plate. Then the absorbance at $570 \mathrm{~nm}$ was measured with an ELISA reader (Ceres UV900c, Bio-Tek Instruments, Windoski, VT, USA). MTT levels are normalised to the number of cells in the scaffolds. As control increasing numbers $(1000,2500,5000,10,000)$ of BMMCs, directly seeded in 96-well plate, were assessed as well.

\section{Scanning Electron Microscopy (SEM) analysis}

For SEM analysis, BMMCs $\left(10^{4}\right.$ cells/well $)$ were cultured on silicate granules or DMB for $72 \mathrm{~h}$ and processed as described previously. Briefly, cells attached to the biomaterials were washed with PBS, fixed in $4 \%$ paraformadehyde/PBS and dehydrated with increasing ethanol percentage (30-90\% in water for 5 minutes and twice with $100 \%$ ethanol for 15 minutes) then treated in Critical Point Dryer (EMITECH K850) and finally sputter coated with platinum-palladium (Denton Vacuum DESKV) and observed with Supra 40 FE-SEM (Zeiss).

\section{Patients}

Twenty patients with osteolytic malignant lesions in different bone segments underwent to clinical, radiological, histopathology evaluation and following surgical resection. All data was recorded on a prefixed proforma (Table 1 and Supplementary Table 1). The lesions were located in 12 patients at the proximal femoral metaphysis, in 4 patients at the proximal tibia, in 3 patients at the proximal humerus and 1 patient at the knee. The histological diagnoses of lesions were $(n=10)$ GCT (Giant cell tumor), $(n=4)$ aneurysmal bone cyst, $(n=3)$ epiphyseal chondroblastoma and $(n=3)$ chondromyxoid fibroma. The mean volume of the lesion, measured with a preoperative computational tomography (CT), was $18.5 \mathrm{~cm}^{2}$ (range 16-24). After surgery all 
Citation: Fazioli F, Colella G, Camerlingo R, Miceli R, Salvatore MGD, et al. (2015) Silicate Granules Preconditioned with Human Bone Marrow Mononuclear Cells Improve Osteogenesis in Bone Sarcoma Patients. J Cancer Sci Ther 7: 321-327. doi:10.4172/1948-5956.1000370

patients were subjected to clinical and radiograph examination every 2 weeks during the distraction phase and every month thereafter until the end of the consolidation phase (one year). Follow-up evaluation was done by using the V.A.S. scale [22,23] and Musculoskeletal Tumour Society (MTS) scoring system [24]. Numerical values from 0 to 5 points were assigned for each of the following 6 categories: pain, function, emotional acceptance, use of supports, walking ability and gait. These values were added, and the functional score was presented as a percentage of the maximum possible score. The results were graded according to the following scale: Excellent- $75 \%$ to $100 \%$; good- $70 \%$ to $74 \%$; moderate- $60 \%$ to $69 \%$; fair- $50 \%$ to $59 \%$ and poor- $-50 \%$. This study was approved by the institutional review board of the author's institution Istituto Nazionale Tumori G Pascale Napoli was performed in accordance with the ethical standards of the 1964 Declaration of Helsinki as revised in 2000. The bone reconstitution stage was determined by clinical and radiograph examination every 2 weeks

\begin{tabular}{|l|l|}
\hline Patients & 20 \\
\hline Age range (18-35) & mean 27 \\
\hline Median B.M.I. $\mathrm{kg} / \mathrm{m}^{2}$ (18.8-25.9) & mean 22.3 \\
\hline Smokers & no \\
\hline Diabetes mellitus patients & no \\
\hline Hypertension patients & no \\
\hline Follow-Up per week (range) & $(2-48)$ \\
\hline Histology & \\
\hline GCT & 10 \\
\hline Aneurysmal Bone Cyst & 4 \\
\hline Epiphyseal Chondroblastoma & 3 \\
\hline Chondromyxoid Fibroma & 3 \\
\hline Tumor site & \\
\hline Femur metaphysis & 12 \\
\hline Tibia & 4 \\
\hline Humerus & 3 \\
\hline Knees & 1 \\
\hline Treatment & \\
\hline Silicate granules & 16 \\
\hline Silicate granules + BMMCs & 4 \\
\hline Lesion area & mean $18.5 \mathrm{~cm}^{2}$ \\
\hline range $\left(16-21 \mathrm{~cm}^{2}\right.$ ) & \\
\hline
\end{tabular}

GCT=giant cells tumor

Table 1: Clinical pathological characteristic of patients. during the distraction phase and the callus type was classified according to the International system [25].

\section{Surgery and preconditioned silicate granules}

Patients were subjected to surgery according to international guidelines under general anesthesia. In the first part of the surgery 20 $\mathrm{ml}$ of autologous bone marrow was aspirated with "Jamshidi" 8-gauge syringe, containing anticoagulant citrate dextrose, from the anterior superior iliac spine. After incision and excision of the biopsy tissue, it was opened a wide bone window through which it was possible to make careful a curettage. Extended curettage was done using a high speed $(7,000 \mathrm{~g}$, Midasrex@) Phenol ( $1 \mathrm{ml}$ of melted phenol mixed with $10 \mathrm{ml}$ of normal saline) as chemical adjuvant in all cases. The cavity was then packed with autologous BMMCs and/or silicate granules (ranging from $10 \mathrm{ml}$ to $40 \mathrm{ml}$ ). The mean ratio between BMMCs and silicate granules was $\left(0.3-1 \times 10^{7}\right.$ BMMCs $/ 10 \mathrm{ml}$ granules Actifuse $\left.{ }^{\oplus}\right)$.

\section{Statistical analysis}

Data are expressed as the mean of \pm standard deviation. Statistical analyses of cell-biology experiments, which were performed in triplicate, were carried with $\mathrm{T}$ student-test analysis $(\mathrm{P}<0.05$ was considered significant).

\section{Results}

To monitor the capability and time of BMMCs to seed on granules, mononuclear fraction was plated on dishes coated with silicate granules or with DBM (see methods). The number of cells present in medium, following 3-time washes, were monitored at different time points during the assay period and reported as percentage of seeding cells (see methods). As shown in Figures 1A, in 30 minutes $90 \%$ of cells adhered to silicate granules showing an exponential progression. In contrast $20 \%$ of BMMCs seeded on DBM after 30 minutes and 5\% in matrigel coated dishes respectively $(\mathrm{P}<0.001$ and $\mathrm{P}<0.05)$. Survival of cells were observed up to day 10 by the MTT assay on each scaffold with active metabolism. No differences were observed in all cases as showed in Figures 1B. Moreover, BMMCs seeded in plate at low density $(1,000$ cells $/ \mathrm{cm}^{2}$ ) gave a spherical phenotype, often accumulated in clusters and were capable to form colonies as shown in Figures 1C. In order to evaluate the fractions of cells with putative regenerative capacities, cells following seeded on silicate granules, were analyzed by flow cytometry in agreement with the International Society for Cell Therapy guidelines [26]. In the analyzed samples, $85 \%$ of the cells co-expressed CD105
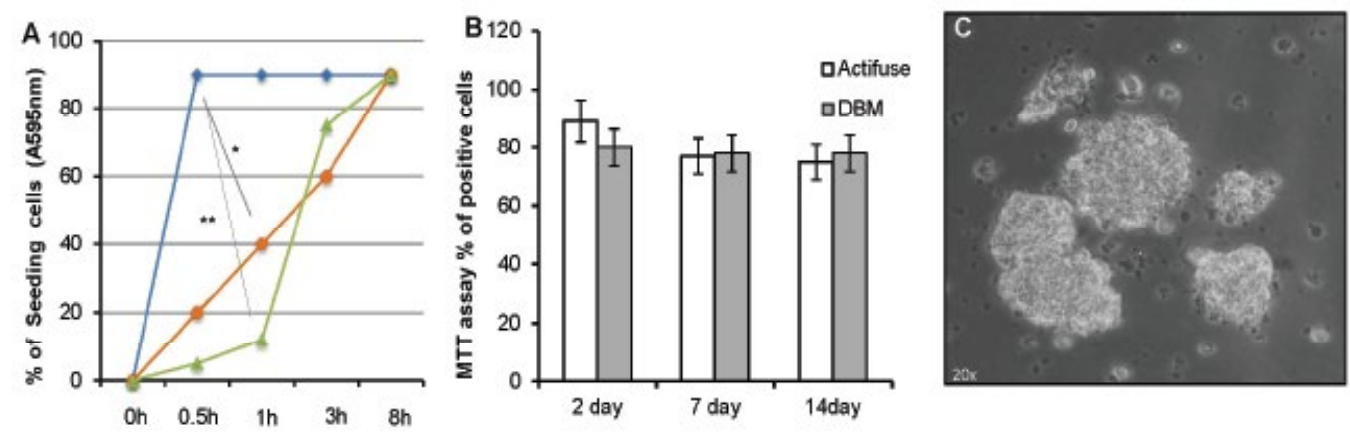

Figure 1: A: Percentage of adhering cells on: silicate granules (indicated with square), DMB (indicated with circle) and matrigel (indicated with triangle) measured by fluorescence intensity at $t=30 \mathrm{~min}, t=1 \mathrm{~h}, t=4 \mathrm{~h}$ and $\mathrm{t}=8 \mathrm{~h}$ of culture times. Statistically significant differences were evident among biomaterials such $* * P<0.001$ silicate granules versus matrigel coated plates and ${ }^{*} P<0.05$ silicate granules versus demineralized bovine bone (DMB). All experiments were performed in triplicated. $B$ : metabolic activity of adhering BMMCs over the time on different surfaces. BMMCs seeded on silicate granules and DMB were cultured over 14 days. Percentage of adhering cells on silicate granules was determined at day 2, day 7 and day 14 (white bars). Number of adhering cells on DMB was determined at same days and indicated with light grey bars. C: Typical growth conformation of stem cells at low density (unit formation colony). 
Citation: Fazioli F, Colella G, Camerlingo R, Miceli R, Salvatore MGD, et al. (2015) Silicate Granules Preconditioned with Human Bone Marrow Mononuclear Cells Improve Osteogenesis in Bone Sarcoma Patients. J Cancer Sci Ther 7: 321-327. doi:10.4172/1948-5956.1000370

and CD90, 89.61\% CD73, and 50.61\% Stro-1 antigens. Cells were negative for hematopoietic antigens CD14, CD11b, CD34, CD19 and CD45. Some representative antigens expression are showed Figure 2. Scanning electron microscopy showed that BMMCs grown on silicate granules had normal morphology and appeared to be well attached to the substrate with long pseudopodia in contact with the extra-cellular matrix (Figures $3 \mathrm{~A}$ and $3 \mathrm{~B}$ ). The amount of debris on the cell surface was low, according to this type of biomaterials. Different behavior was registered towards DBM attached cells showed a low number of shorter pseudopodia and greater amount of cells in natural bone niche (Figures $3 \mathrm{C}$ and 3D). For in vivo study, we analyzed twenty bone sarcoma patients whose the clinical pathological characteristics are reported in Table 1 and supplementary Table 1 . At the clinical examination, the patients presented spontaneous pain localized to the site of the lesion with functional analgesic limitation. The pain was exacerbated by acupressure and it was possible to appreciate the tumefaction of the tumor. The mean age of patients was 27 years, tumors histology were different and localized in different sites as indicated in Table 1 and supplementary Table 1 . The radiological examination, before surgical resection of tumor showed the presence of a large osteolytic area with mean of $18.5 \mathrm{~cm}^{2}$. A representative cases are showed in Figure $4 \mathrm{~A}$ and $4 \mathrm{D}$. A total of 16 patients underwent to reconstructive curettage and filling of the lesion site with silicate granules alone. The Figure $4 \mathrm{~A}$ reported the case of patient affect from chondroblastoma of knee filled with silicate granules alone. After 15 days, the patient showed a callus shape at $\mathrm{X}$-rays examination (Figure $4 \mathrm{~B}$ and $4 \mathrm{C}$ ) no complication were reported
(Table 2) and the functional outcome following Musculoskeletal Tumor Society score (MSTS) was of $60 \%$ (Tables 2 and 3). The Figure 4D reported a representative patient subjected to curettage and filling of humerus lesion with silicate granules combined with BMMCs. The $\mathrm{X}$-rays examination of bone cavity revealed the presence of callus after two weeks (Figure $4 \mathrm{E}$ and $4 \mathrm{~F}$ ). Silicate granules introduced into the humerus cavity showed low material around the periphery of the pores indicated bone formation within the marrow cavity. The outcome at two weeks of humerus filled with silicate granules and BMMCs was $78 \%$ by MSTS score compared to $58 \%$ of humerus filled with silicate granules alone (Table 3 and Supplementary Table 1). After 4 weeks all patients treated had bone cavities completely occupied by bone callus as reported in Tables 2 and 3. None patients showed necrosis or infection of lesions (Table 2) that required additional surgery of bone.

\section{Clinical outcome}

According to the V.A.S. evaluation system there were no significant differences at 2, 4, 48, weeks, after the surgery. According to the MSTS evaluation system, after the surgery, we found that patients treated with curettage and filling of the defects with silicate granules and BMMCs had mean of MSTS score of $82 \%$ (Table 2 and Table 3). In contrast patients treated silicate granules alone the mean of MSTS score was $60 \%$. During the follow up (48 weeks) none patients develop infection or hematoma that requires surgical revision neither necrosis. The encouraging preliminary results are reported in Tables 2 and 3.
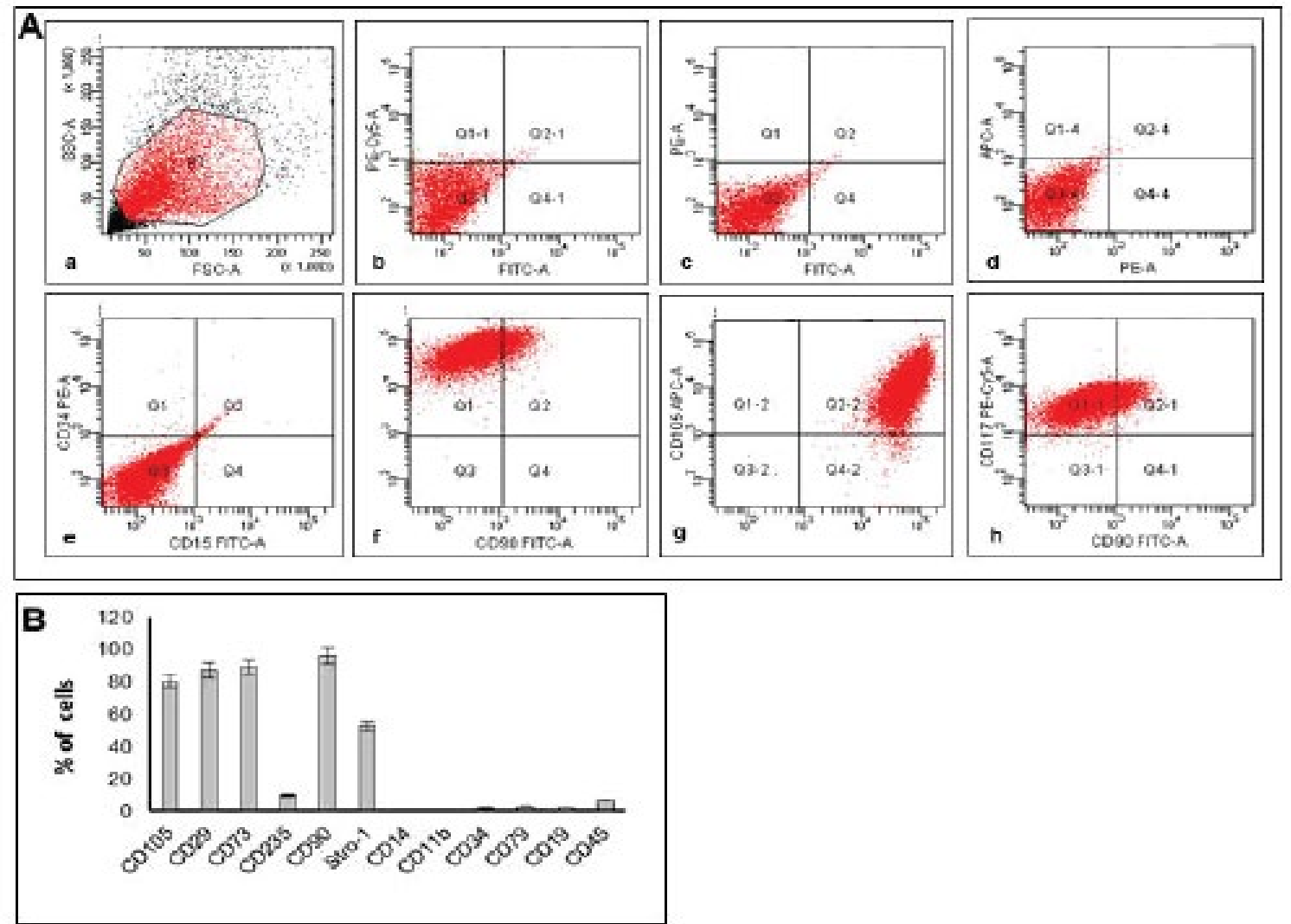

Figure 2: A: Surface antigenic profile of BMMCs obtained from bone marrow aspirates at the first culture passage analyzed by FACS. a: BMMCs control. b-d: FACS setting with fluorochromes. e: CD34 and CD15 surface antigens co-expression characterization. f: $85.7 \%$ of BMMCs expressed CD90 antigen. g: $95.1 \%$ of cells expressed CD105. h: co-expression of CD90 and CD117 antigens. B: percentage of positive cells to other antigens such as CD14, and CD11b as indicated. 
Citation: Fazioli F, Colella G, Camerlingo R, Miceli R, Salvatore MGD, et al. (2015) Silicate Granules Preconditioned with Human Bone Marrow Mononuclear Cells Improve Osteogenesis in Bone Sarcoma Patients. J Cancer Sci Ther 7: 321-327. doi:10.4172/1948-5956.1000370
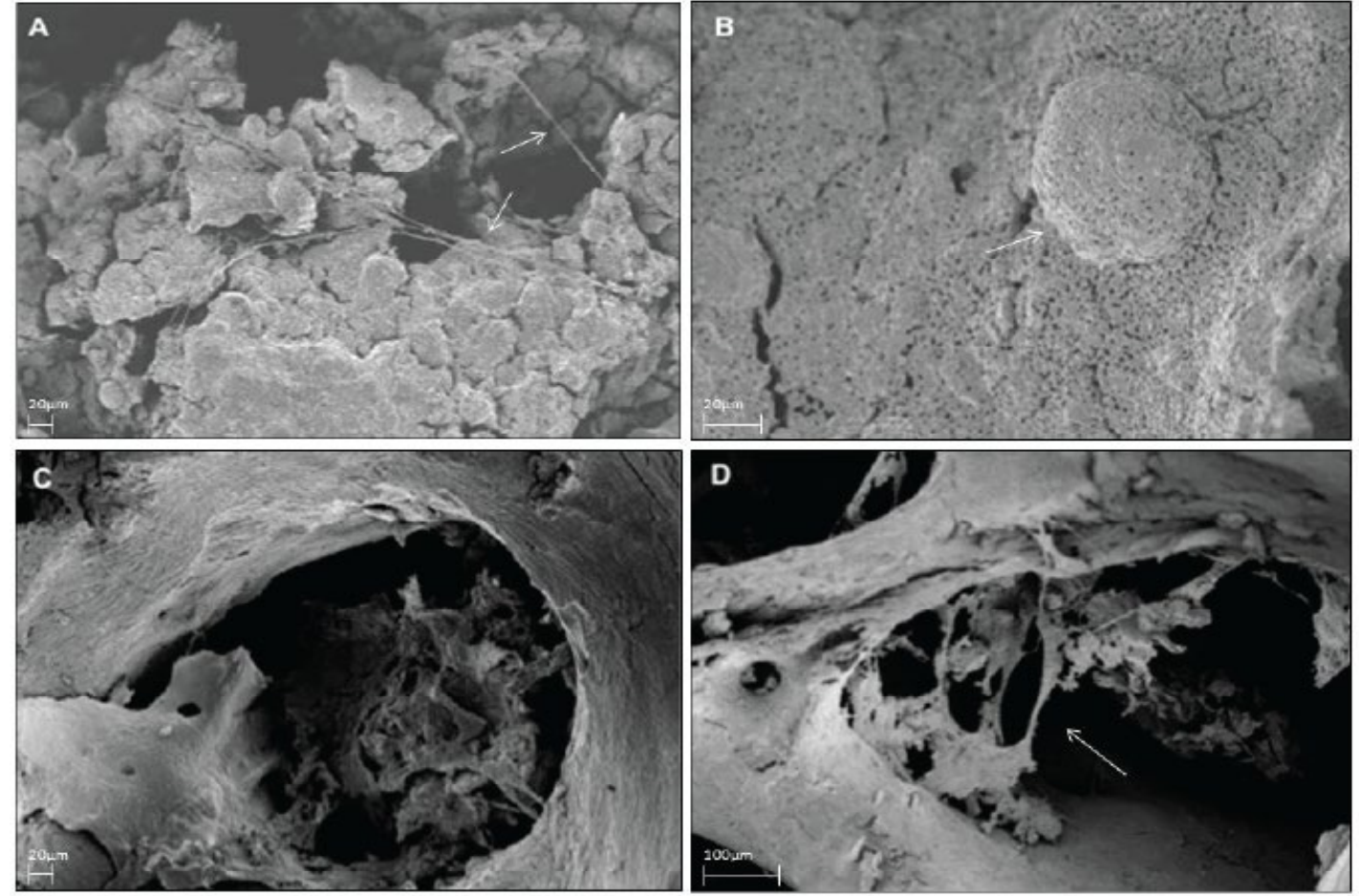

Figure 3: A and B: SEM morphology analysis of BMMCs cultured on silicate granules. Cells appear to be well attached to the substrate with several pseudopodias and cytoplasmic extroflessions as indicated by arrows. A large amount of debris is present on the surface of cells grown on silicate granules. C and D: SEM morphology analysis of BMMCs cultured in presence of DMB. Cells form multi-aggregate localized into bone cavity with short pseudopodias. The arrows indicated pseudopodias.
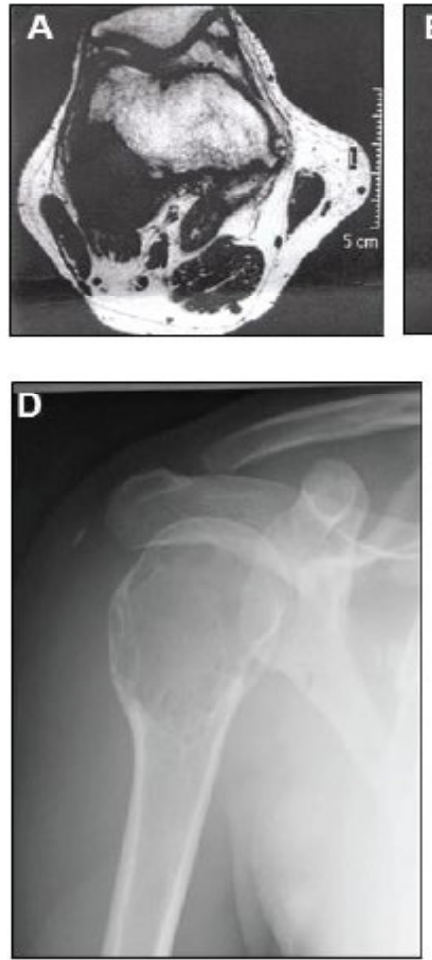
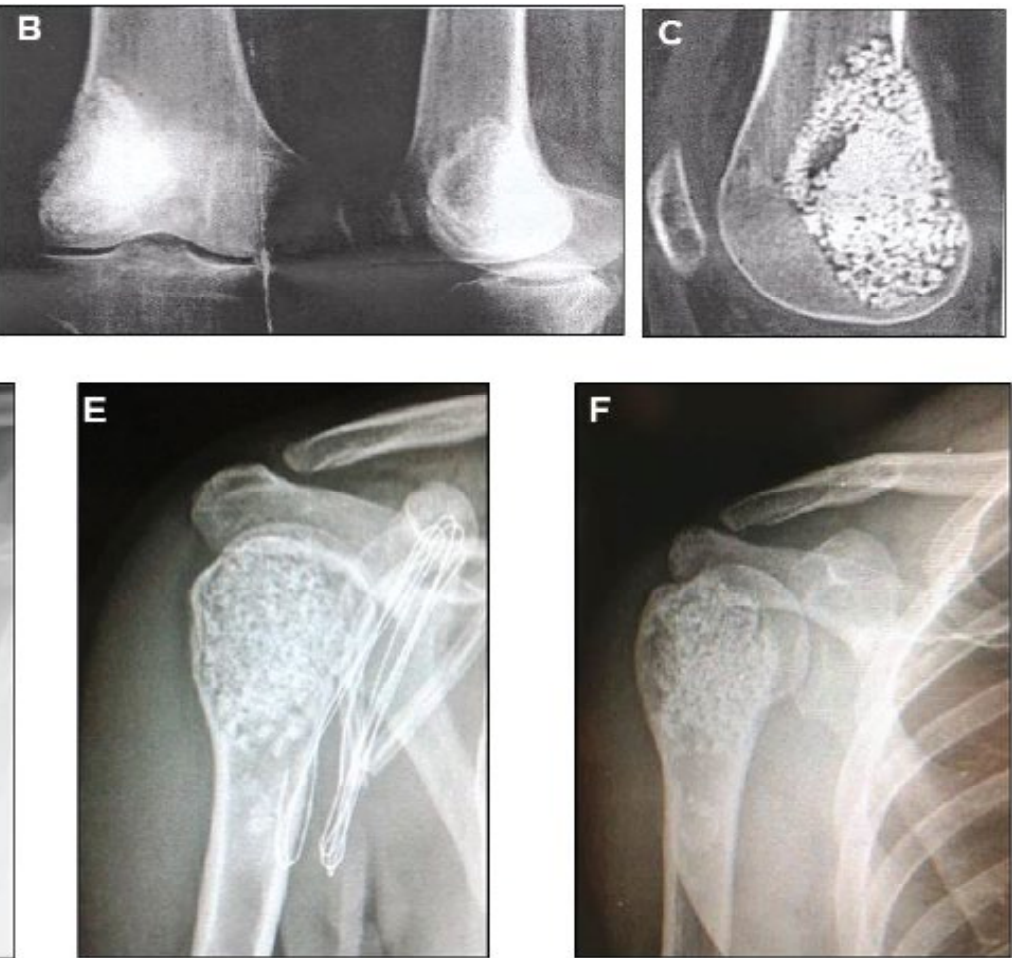

Figure 4: A: Pre-operative X-rays of the Knee. B: X-rays after 2 weeks post-surgery. Knee filled with silicate granules alone. C: X-rays follow-up at 30 days. Knee treated with granules silicate alone. D: Pre-operative X-rays of aneurysmal bone cyst of proximal humerus. E: X rays after 2 weeks post-surgery. The humerus cavity was filled with BMMCs and silicate granules. F: X-rays follow-up at 30 days of the humerus (treated with BMMCs and silicate granules). 


\begin{tabular}{|l|c|c|c|}
\hline \multirow{2}{*}{ Complications } & \multicolumn{3}{|c|}{ Follow-up } \\
\cline { 2 - 4 } & $\mathbf{2}$ weeks & $\mathbf{4}$ weeks & $\mathbf{4 8}$ weeks \\
\hline Nr. Late Seroma formation & 0 & 0 & 0 \\
\hline Infection & 0 & 0 & 0 \\
\hline Hematoma self-limiting & 1 & 0 & 0 \\
\hline Hematoma requiring surgical revision & 0 & 0 & 0 \\
\hline Partial skin flap necrosis & 1 & 1 & 1 \\
\hline NAC necrosis & 0 & 0 & 0 \\
\hline $\begin{array}{l}\text { Overall feelings about bone } \\
\text { reconstruction (V.A.S. scale) }\end{array}$ & 6,81 (medium) & 8 (good) & 8 (good) \\
\hline Simmetry (V.A.S. scale) & 6,71 (good) & 7 (good) & 9 (excellent) \\
\hline
\end{tabular}

Table 2: Patients follow-up.

\begin{tabular}{|c|c|c|c|c|}
\hline & \multirow[t]{2}{*}{ Tumor site } & \multirow[t]{2}{*}{ Treatment } & \multicolumn{2}{|c|}{ MSTS } \\
\hline & & & 2 weeks & 4 weeks \\
\hline \multirow[t]{3}{*}{ Upper Limb } & 1 humerus & Silicate granules & $58 \%$ & $74 \%$ \\
\hline & 1 humerus & Silicate granules+BMMCs & $81 \%$ & $90 \%$ \\
\hline & 1 humerus & Silicate granules+BMMCs & $78 \%$ & $88 \%$ \\
\hline \multirow[t]{5}{*}{ Lower Limb } & 1 knees & Silicate granules & $59 \%$ & $72 \%$ \\
\hline & 11 Femur & Silicate granules & $62 \%$ & $75 \%$ \\
\hline & 1 Femur & Silicate granules+BMMCs & $72 \%$ & $76 \%$ \\
\hline & 3 Tibia & Silicate granules & $51 \%$ & $65 \%$ \\
\hline & 1 Tibia & Silicate granules+BMMCs & $85 \%$ & $92 \%$ \\
\hline
\end{tabular}

Table 3: Musculoskeletal tumour society functional outcome score (msts) into upper and lower limb.

\section{Discussion}

Research in tissue engineering is focused on finding new approaches for bone regeneration that optimize results by seeding BMMCs on a porous ceramic scaffold. Several orthopedic studies in large animals emphasize the difference between the healing of sites treated with scaffolds loaded with BMMCs compared to scaffolds without BMMCs $[27,28]$. Moreover, silicate granules has shown promising results, in bone regenerative therapy with fast bone apposition rates [28]. In human, ex vivo studies have investigated biocompatibility between stem cells and synthetic material for bone regeneration [29]. In patients progress has been made in ligament regeneration using BMMCs [30] and several trials are ongoing [31]. Our preliminary study utilizes, for the first time, a combination of silicate granules with BMMCs to improve bone repair and to provide immediate support for large tumor cavities in human [32]. To characterize the behavior in vitro of silicate granules with bone marrow aspirates from orthopedic patients we cultured BMMCs in close contact with selected granules. Viability assays demonstrated that the biomaterial did not elicit any cytotoxic effects. The positive charge present on the silicate material promoted cell adhesion in a few minutes [29]. Additionally, fluocytometry analysis indicated that most of adhered cells were mesenchymal with high regenerative potential. Findings from SEM analysis showed a well-organized cytoskeleton architecture and long cytoplasmic bridges between cells and substrate. The ability of BMMCs to contact a large area of the silicate material is an important indicator to evaluate long-term healing and stability of silicate granules in human bone. Our preliminary findings demonstrated that the combinatory use of autologous BMMCs and silicate granules improved bone regeneration within lesion cavity detected as formation of callus and a mean MSTS of $85 \%$ at two week's follow-up. Further studies will be necessary to determine why and under which conditions the new bone wall develops and new cartilage grows on regenerated bone.

\section{Conclusions}

In this preliminary study, we investigated bone reconstruction using silicate granules in combination with autologous BMMCs. Patients with a small and medium-sized bone cavity (mean $18.5 \mathrm{~cm}^{2}$ ) had successful single-stage outcomes. In summary, post-surgery use of silicate granules in combination with autologous BMMCs to fill the bone lesion appears to improve rapidity of bone integrity reconstruction. However, a larger study cohort and longer follow-up times are required to identify additional predictors and indications.

\section{Acknowledgments}

The authors wish to acknowledge: Dr.ssa Claudia San Domenico for her support for critical reading of the manuscript and Dr.ssa Marcella Cammarota for Scanning electron microscopy of the cells.

\section{Consent}

Written informed consent was obtained from the patients for the publication of this report and any accompanying images.

\section{Competing interests}

All the Authors disclose that in the past five years they do not received reimbursements, fees, funding, or salary from an organization that may in any way gain or lose financially from the publication of this manuscript, either now or in the future. All the Authors disclose that they do not received reimbursements, fees, funding, or salary from an organization that holds or has applied for patents relating to the content of the manuscript. All the Authors disclose that they do not have any other financial competing interests. All the Authors disclose that they do not have any non-financial competing interests (political, personal, religious, ideological, academic, intellectual, commercial or any other) to declare in relation to this manuscript.

\section{Ethical standards}

The patients have given their informat consent prior to being included in the study. The study was authorized by the local ethical commitee from Istituto G Pascale (Naples) Italy and was performed in accordance with the ethical standards of the 1964 Declaration of Helsinki as revised in 2000.

\section{Author's contributions}

GP, RC, and RM performed in vitro experiment and FACS analysis. FF He was the senior surgeon for all the surgical procedures reported in the article. He has made fundamental contributions to conception and design, acquisition of data, critical analysis and interpretation of data. He was the supervisor for all the research group at any time of surgery and follow-up. He has given final approval of the version to be published. He agree to be accountable for all aspects of the work in ensuring that questions related to the accuracy or integrity of any part of the work are appropriately investigated and resolved. He has given final approval of the version to be published. FDN He has been involved in drafting the manuscript and revising it critically for important intellectual content. RDV assistant surgeon for all the surgical procedures reported in the article. GC He has participated sufficiently in the work to take public responsibility for appropriate portions of the content. $\mathrm{He}$ was FF assistant surgeon for all the surgical procedures reported in the article. MDS He has participated sufficiently in the work to take public responsibility for appropriate portions of the content. He has given final approval of the version to be published. He was FF assistant surgeon for all the surgical procedures reported in the article. All authors read and approved the final manuscript.

\section{References}

1. Muramatsu K, Ihara K, Yoshida K, Tominaga Y, Hashimoto T, et al. (2013) Musculoskeletal sarcomas in the forearm and hand: standard treatment and microsurgical reconstruction for limb salvage. Anticancer Res 33: 4175-4182.

2. Van Heest A, Swiontkowski M (1999) Bone-graft substitutes. Lancet 353 Supp 1: SI28-29.

3. Wolff D, Goldberg VM, Stevenson S (1994) Histomorphometric analysis of the repair of a segmental diaphyseal defect with ceramic and titanium fibermetal implants: effects of bone marrow. J Orthop Res 12: 439-446.

4. Gogolewski S, Jovanovic M, Perren SM, Dillon JG, Hughes MK (1993) Tissue response and in vivo degradation of selected polyhydroxyacids: polylactides (PLA), poly(3-hydroxybutyrate) (PHB), and poly(3-hydroxybutyrate-co-3hydroxyvalerate) (PHB/VA). J Biomed Mater Res 27: 1135-1148.

5. Berger G, Gildenhaar R, Ploska U (1995) Rapid resorbable, glassy crystalline materials on the basis of calcium alkali orthophosphates. Biomaterials 16 1241-1248. 
Citation: Fazioli F, Colella G, Camerlingo R, Miceli R, Salvatore MGD, et al. (2015) Silicate Granules Preconditioned with Human Bone Marrow Mononuclear Cells Improve Osteogenesis in Bone Sarcoma Patients. J Cancer Sci Ther 7: 321-327. doi:10.4172/1948-5956.1000370

6. Daculsi G (1998) Biphasic calcium phosphate concept applied to artificial bone implant coating and injectable bone substitute. Biomaterials 19: 1473-1478.

7. Hardenbrook MA, Lombardo S. (2006) Silicate-substituted calcium phosphate as a bone void filler after kyphoplasty in a young patient with multiple compression fractures due to osteogenesis imperfecta variant: case report. Neurosurg Focus 6:1-5

8. Chen QZ, Thompson ID, Boccaccini AR (2006) 45S5 Bioglass-derived glassceramic scaffolds for bone tissue engineering. Biomaterials 27: 2414-2425.

9. Carson JS, Bostrom MP (2007) Synthetic bone scaffolds and fracture repair. Injury 38 Suppl 1: S33-37.

10. Winter M, Griss P, de Groot K, Tagai H, Heimke G, et al. (1981) Comparative histocompatibility testing of seven calcium phosphate ceramics. Biomaterials 2: 159-160.

11. Henrich D, Verboket R, Schaible A, Kontradowitz K, Oppermann E, Brune JC, et al. (2015) Characterization of bone marrow mononuclear cells on biomaterials for bone tissue engineering in vitro. Biomed Res Int 2015: 762407

12. Asatrian G, Pham D, Hardy WR, James AW, Peault B (2015) Stem cell technology for bone regeneration: current status and potential applications. Stem Cells Cloning 8: 39-48.

13. Lucarelli E, Fini M, Beccheroni A, Giavaresi G, Di Bella C, et al. (2005) Stroma stem cells and platelet-rich plasma improve bone allograft integration. Clin Orthop Relat Res : 62-68.

14. Minamide A, Yoshida M, Kawakami M, Yamasaki S, Kojima H, et al. (2005) The use of cultured bone marrow cells in type I collagen gel and porous hydroxyapatite for posterolateral lumbar spine fusion. Spine (Phila Pa 1976) 30: 1134-1138.

15. Koyanagi H, Ae K, Maehara H, Yuasa M, Masaoka T, et al. (2013) Massive bone reconstruction with heat-treated bone graft loaded autologous bone marrow-derived stromal cells and $\beta$-tricalcium phosphate composites in canine models. J Orthop Res 8:1308-1316.

16. Cao L, Liu X, Liu S, Jiang Y, Zhang X, et al. (2012) Experimental repair of segmental bone defects in rabbits by angiopoietin-1 gene transfected MSCs seeded on porous $\hat{I}^{2}$-TCP scaffolds. J Biomed Mater Res B Appl Biomater 100: 1229-1236.

17. Quarto R, Mastrogiacomo M, Cancedda R, Kutepov SM, Mukhachev V, et al (2001) Repair of large bone defects with the use of autologous bone marrow stromal cells. N Engl J Med 344: 385-386.

18. Moran JM, Pazzano D, Bonassar LJ (2003) Characterization of polylactic acid- polyglycolic acid composites for cartilage tissue engineering. Tissue Eng 9 63-70.

19. Gan Y, Dai K, Zhang P, Tang T, Zhu Z, et al. (2008) The clinical use of enriched bone marrow stem cells combined with porous beta-tricalcium phosphate in posterior spinal fusion. Biomaterials 29: 3973-3982.

20. Petite H, Viateau V, Bensaïd W, Meunier A, de Pollak C, et al. (2000) Tissueengineered bone regeneration. Nat Biotechnol 18: 959-963.

21. Pittenger MF, Mackay AM, Beck SC, Jaiswal RK, Douglas R, et al. (1999) Multilineage potential of adult human mesenchymal stem cells. Science 284 143-147.

22. Tian LF, Yuan YS, Li EH, Wang L (2015) Different preoperative approaches for acute lumber spinal fractures. Int J Clin Exp Med 8: 9979-9984.

23. De Vita R, Buccheri EM, Pozzi M, Zoccali G (2014) Direct to implant breast reconstruction by using SERI, preliminary report. J Exp Clin Cancer Res 33: 78.

24. Kristensen MT, Foss NB, Kehlet H (2009) Factors with independent influence on the 'timed up and go' test in patients with hip fracture. Physiother Res Int 14: 30-41.

25. Li R, Saleh M, Yang L, Coulton L (2006) Radiographic classification of osteogenesis during bone distraction. J Orthop Res 24: 339-347.

26. Dominici M, Le Blanc K, Mueller I, Slaper-Cortenbach I, Marini F, et al. (2006) Minimal criteria for defining multipotent mesenchymal stromal cells. The International Society for Cellular Therapy position statement. Cytotherapy 4 315-331.

27. Zhu L, Liu W, Cui L, Cao Y (2006) Tissue-engineered bone repair of goat femur defects with osteogenically induced bone marrow stromal cells. Methods enzymol 420: 362-380.

28. Yuan J, Cui L, Zhang WJ, Liu W, Cao Y (2007) Repair of canine mandibula bone defects with bone marrow stromal cells and porous -tricalciumphosphate. Biomaterials 6: 1005-1013.

29. Rosset P, Deschaseaux F, Layrolle P3 (2014) Cell therapy for bone repair Orthop Traumatol Surg Res 100: S107-112.

30. Bait C, Denti M, Prospero E, Quaglia A, Orgiani A, et al. (2015) Posterior cruciate ligament reconstruction with "all-inside" technique: a technical note. Muscles Ligaments Tendons J 4: 467-470.

31. Dawson JI, Kanczler J, Tare R, Kassem M, Oreffo RO (2014) Concise review: bridging the gap: bone regeneration using skeletal stem cell-based strategies where are we now? Stem Cells 32: 35-44.

32. Labs K, Perka C, Schmidt RG (2001) Treatment of stages 2 and 3 giant-cel tumor. Arch Orthop Trauma Surg 121: 83-86. 